\title{
Síntese e Caracterização do Polímero Energético Metil Azoteto de Glicidila (GAP) Via Análises Instrumentais
}

\author{
Jairo Sciamareli, Jorge R. da Costa, Marta F. K. Takahashi, Milton F. Diniz, Vera L. Lourenço, Luis H. David \\ Divisão de Química, IAE, CTA \\ Koshun Iha \\ Instituto Tecnológico de Aeronáutica, ITA \\ Miriam H. Miyano, Carlos Ferreira \\ Cognis Brasil Ltda
}

\begin{abstract}
Resumo: na área aeroespacial é constante a pesquisa em busca de novos materiais que permitam um melhor desempenho de foguetes, mísseis e explosivos. Entre outros, compostos mais energéticos, de maior estabilidade térmica e/ou química, menos agressivos ao meio ambiente, de menor custo e de mais fácil manuseio. Um destes materiais é o metil azoteto de glicidila (GAP), que pode ser utilizado para produzir propelentes mais energéticos e/ou sem fumaça, bem como composições explosivas de baixa sensibilidade. O GAP foi sintetizado e caracterizado por análises FT-IR, elementar, cromatográfica de permeação de gel e térmica.
\end{abstract}

Palavras-chave: Polímero energético, GAP, propelente compósito.

\section{Characterization of Energetic Methyl Glycidyl Azide Polymer}

\begin{abstract}
To obtain more energetic, safe propellants, with improved performance, better mechanical properties and low cost, new materials have been researched and tested. One of these materials is Methyl Glycidyl Azide Polymer (GAP). It can be used to make energetic and/or smokeless propellants as well as composite explosives with low sensitivity. GAP was synthesized and characterized by FT-IR, elemental analysis, gel permeation chromatography (GPC) and thermal analysis.
\end{abstract}

Keywords: Energetic polymers, GAP, composite propellants.

\section{Introdução}

Propelente sólido compósito é uma mistura complexa e, ao mesmo tempo, estável de oxidantes e redutores que, quando ignitado, queima de uma maneira controlada com emissão gasosa de espécies de baixa massa molar ${ }^{[1]}$.

Nos últimos anos tem sido constante a busca por novos materiais que possam ser utilizados em propelentes. Buscam-se, entre outras características, materiais mais energéticos, de maior estabilidade térmica e/ou química, menos agressivos ao meio ambiente, de menor custo, de manuseio mais fácil, que permitam um melhor desempenho de foguetes, mísseis e explosivos.

O Metil Azoteto de Glicidila (GAP) corresponde a esta expectativa. É um polímero que apresenta em cada unidade de repetição um grupo azida $\left(\mathrm{N}_{3}\right)$ e grupos $\mathrm{OH}$ nas extremidades da cadeia, sendo, portanto bifuncional. O GAP pode sofrer reações de cura por meio da combinação destes grupos $\mathrm{OH}$ com grupos diisocianato presentes em compostos como isoforona diisocianato (IPDI) e tolueno diisocianato
(TDI). Deste modo, a funcionalidade do polímero é um importante parâmetro nas propriedades mecânicas requeridas por um elastômero curado ${ }^{[2]}$. A presença de grupos azida torna o produto energético, tendo o produto calor de formação positivo, da ordem de $+975 \mathrm{~kJ} / \mathrm{kg}$ a $293 \mathrm{~K}^{[3]}$. Assim sendo, ele pode ser empregado na produção de propelentes energéticos e/ou propelentes "sem fumaça", também conhecidos como "smokeless".

De modo geral, o GAP é obtido em duas etapas ${ }^{[4]}$ : primeiro obtém-se o intermediário poliepicloridrina $(\mathrm{PECH})$ e com este, na etapa seguinte, obtém-se o GAP.

Da primeira etapa de síntese participam, obrigatoriamente, a epicloridrina $(\mathrm{ECH})$, um iniciador, de preferência um diálcool com grupos $\mathrm{OH}$ terminais e um catalisador do tipo ácido de Lewis. Por meio da reação de abertura do anel epóxi da epicloridrina ocorre o crescimento da cadeia. A alta concentração de epicloridrina no meio reacional favorece a reação indesejada de formação de cíclicos ${ }^{[5]}$.

Em uma segunda etapa, a poliepicloridrina obtida reage com azida de um metal, normalmente azida de sódio, para

Autor para correspondência: Jairo Sciamareli, Instituto de Aeronáutica e Espaço, Divisão de Química (AQI), Pça Mal. do Ar Eduardo Gomes 50, VI das Acácias, CEP: 12228-904, São José dos Campos, SP, Brasil. E-mail: sciamareli@iae.cta.br 
formar o GAP. Essa reação ocorre com o uso de solventes apróticos e apolares.

No Brasil, o Comando-Geral de Tecnologia Aeroespacial (CTA) por meio do Instituto de Aeronáutica e Espaço (IAE) busca a mais moderna tecnologia em foguetes e veículos lançadores de satélites, propelentes sólidos compósitos e seus aditivos e proteções térmicas. Dentro deste contexto se inclui a obtenção e caracterização do GAP por meio de análises instrumentais.

\section{Experimental}

\section{Reagentes}

Epicloridrina marca Fluka, grau de pureza 98\%, dicloroetano marca Aldrich, grau de pureza 99\%, tetracloreto de estanho $\left(\mathrm{SnCl}_{4}\right)$ marca Aldrich, solução 1 molar em diclorometano, ácido trifluoracético marca Aldrich, 99\% de pureza, butanodiol 1,4 marca Carlo Erba, grau de pureza 99\%, dimetil formamida (DMF) marca Merck, grau de pureza 99,5\% e azida de sódio marca Vetec, grau de pureza 99\%. Os reagentes foram utilizados como recebidos, exceção à epicloridrina e o dicloroetano que foram tratados com sulfato de cálcio e posteriormente submetidos à destilação para remoção de traços de umidade.

\section{Obtenção da poliepic/oridrina}

Para obter a poliepicloridrina, foram utilizados como catalisador o tetracloreto de estanho anidro $\left(\mathrm{SnCl}_{4}\right)$, e o ácido trifluoracético como co-catalisador, butanodiol 1,4 como iniciador, além de epicloridrina e dicloroetano. A escolha de butanodiol 1,4 se deveu ao fato que diálcoois inferiores favorecem a formação de cíclicos em detrimento do crescimento da cadeia ${ }^{[5]}$.

Os reagentes, com exceção da epicloridrina, foram colocados em um balão, homogeneizados e aquecidos a $70^{\circ} \mathrm{C}$ sob agitação constante. A epicloridrina foi adicionada aos outros reagentes, gota a gota, por meio do funil de adição, sendo mantida a temperatura. Após o término da adição, o aquecimento do sistema reacional prosseguiu por mais uma hora ${ }^{[6]}$.

Ao final desta etapa, após o resfriamento da solução, foi adicionado água e o sistema agitado por cerca de 15 minutos. A seguir, a fase orgânica, a qual continha o produto desejado, e a fase aquosa foram separadas, com descarte da fase aquosa. Este procedimento foi repetido por mais 4 vezes.

Por fim, a fase orgânica foi levada a um aparelho do tipo evaporador rotativo para extração do dicloroetano.

\section{Obtenção do GAP}

Praticamente com os mesmos equipamentos utilizados na síntese da poliepicloridrina, foi montado um sistema para a obtenção do GAP, sendo utilizado ainda, gás nitrogênio seco para a ocorrência de atmosfera inerte. A poliepicloridrina, solubilizada em dimetil formamida (DMF), foi homogeneizada

$$
\begin{aligned}
& 2 \mathrm{n}_{2} \mathrm{C}-\mathrm{CH}-\mathrm{CH}_{2} \mathrm{Cl}+\mathrm{HO}-\mathrm{R}-\mathrm{OH} \longrightarrow \\
& \mathrm{H}\left(\underset{\mathrm{O}}{\mathrm{O}-\mathrm{CH}-\mathrm{CH}_{2}}-\mathrm{O}-\mathrm{R}-\mathrm{O}-\left(\begin{array}{l}
\mathrm{CH}_{2}-\underset{\mathrm{CH}}{\mathrm{C}}-\mathrm{O} \\
\mathrm{CH}_{2} \mathrm{Cl}
\end{array}\right)_{\mathrm{n}}^{\mathrm{H}}\right.
\end{aligned}
$$

(a)
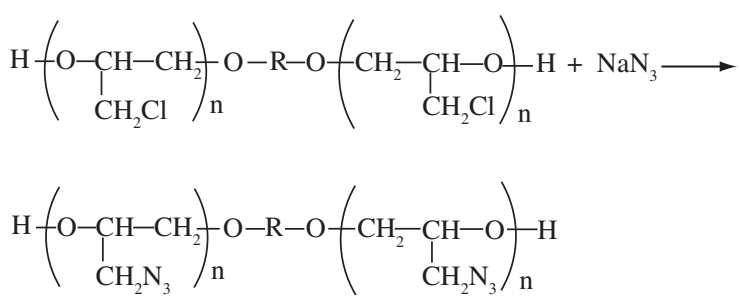

(b)

Figura 1. a) Reação de formação da poliepicloridrina; e b) reação de formação do GAP.

e aquecida, quando foi então, adicionada a azida de sódio. A temperatura foi elevada e mantida por cerca de uma hora.

A solução resultante foi filtrada em um funil raiado equipado com papel de filtro para a separação do resíduo sólido resultante da reação, cloreto de sódio e o excesso de azida de sódio e, a seguir, transferida para um balão com o qual foi montado um sistema de destilação simples para extração do solvente orgânico, sob atmosfera reduzida e com aquecimento.

Ao GAP assim obtido foi adicionado diclorometano e a solução resultante foi agitada por 10 minutos. A seguir, foi adicionada água destilada a $50{ }^{\circ} \mathrm{C}$ e a solução foi novamente agitada por mais 20 minutos e transferida para um funil de separação. Após a separação das fases, a fase orgânica foi recolhida e este processo de lavagem com água foi repetido por mais duas vezes ${ }^{[7]}$.

Ao final, a fase orgânica foi levada a um aparelho evaporador rotativo, onde o diclorometano foi extraído sob pressão reduzida, restando no balão o produto desejado, o GAP, que apresentava um aspecto viscoso e de cor âmbar, tal como descrito em literatura ${ }^{[8]}$.

A Figura 1 ilustra as etapas de síntese: a) obtenção da poliepicloridrina; e b) obtenção do GAP.

\section{Análise instrumental}

A PECH e o GAP obtidos foram submetidos à análise FTIR, como filmes líquidos, em um espectrofotômetro SPECTRUM 2000 - Perkin-Elmer, de acordo com as condições básicas: região $4000-400 \mathrm{~cm}^{-1}$, resolução $4 \mathrm{~cm}^{-1}$, ganho 1, 40 varreduras.

Para as análises térmicas foi utilizado o aparelho Analisador Térmico TA2100 TA Instruments, com os módulos DSC-910 e TGA-951. A análise termogravimétrica (TGA) foi feita em atmosfera de ar sintético a partir da temperatura ambiente até se atingir massa constante, à razão de aquecimento de $10{ }^{\circ} \mathrm{C} / \mathrm{min}$. A análise por calorimetria exploratória 
diferencial (DSC) foi conduzida sob atmosfera de nitrogênio a partir de -100 a $50{ }^{\circ} \mathrm{C}$ a $10{ }^{\circ} \mathrm{C} / \mathrm{min}$.

A análise elementar foi feita em um aparelho Elemental Analyzer CHN2400 Perkin Elmer, com fluxo de oxigênio a $925^{\circ} \mathrm{C}$.

A determinação da massa molecular foi feita por meio de análise de cromatografia de permeação de gel (GPC), utilizando um sistema PL-GPC210 da Polymer Labs, tendo tetrahidrofurano (THF) como fase móvel, concentração de $20 \mathrm{mg} / 10 \mathrm{~mL}$ de amostra, detector RI- índice de refração e temperatura de $30^{\circ} \mathrm{C}$.

\section{Resultados}

A poliepicloridrina, obtida sob a forma de líquido levemente viscoso e translúcido, de cor palha claro, se caracteriza pela unidade repetitiva $-\mathrm{CH}\left(\mathrm{CH}_{2} \mathrm{Cl}\right)-\mathrm{CH}_{2}-\mathrm{O}-$. Em termos espectrofotométricos a absorção mais característica desse polímero é a ligação éter $(\mathrm{C}-\mathrm{O}-\mathrm{C})$, pois, as demais absorções, $\mathrm{CH}, \mathrm{CH}_{2}, \mathrm{CH}_{2} \mathrm{Cl}$, esta última aparecendo em $748 \mathrm{~cm}^{-1}$, também ocorrem nos materiais de partida e, portanto, não são úteis para caracterizar o produto final, ou seja, a PECH. A ligação éter ocorre em torno de $1114 \mathrm{~cm}^{-1}$. O espectro FT-IR da PECH obtida é mostrado na Figura 2.

No espectro FT-IR do GAP, Figura 3, a absorção devido ao estiramento de grupos $\mathrm{CH}_{2} \mathrm{Cl}$, em torno de $748 \mathrm{~cm}^{-1}$ da PECH, desaparece, como esperado, e em contrapartida surgem absorções decorrentes da azidometilação, em torno de 2100 e $1281 \mathrm{~cm}^{-1}$, devido aos estiramentos assimétrico

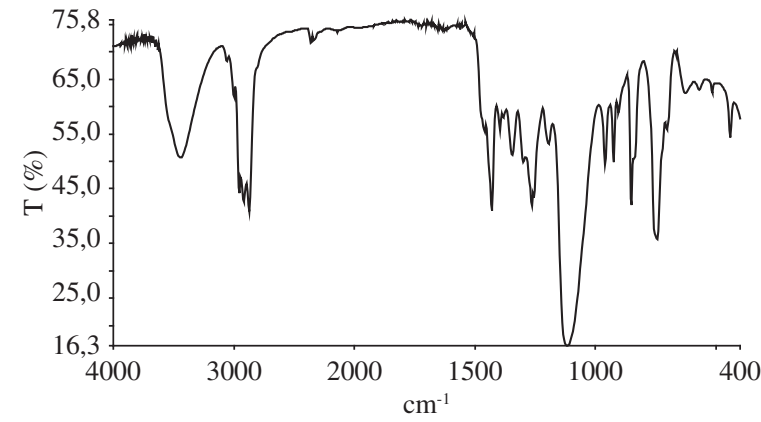

Figura 2. Espectro FT-IR da poliepicloridrina.

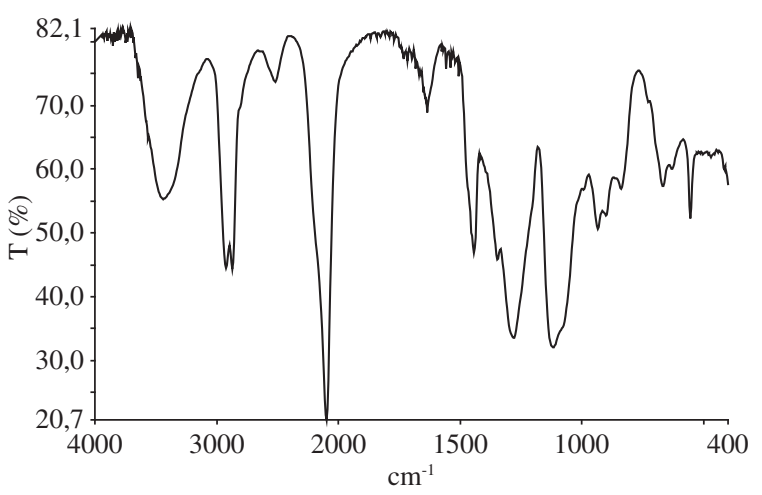

Figura 3. Espectro FT-IR do GAP. e simétrico da ligação $\mathrm{CH}_{2}-\mathrm{N}_{3}$. As absorções em 1118 e $1119 \mathrm{~cm}^{-1}$ e em 3367 e $3446 \mathrm{~cm}^{-1}$, devidas as ligações éter da cadeia principal e aos grupos hidroxila terminais, originalmente da $\mathrm{PECH}$, continuam presentes neste espectro. Isto era esperado uma vez que a estrutura principal continua a mesma e são concordantes com o observado na literatura $^{[9]}$.

Por meio da análise DSC foi obtida a transição vítrea, a qual é evidenciada por um desnível endotérmico da linha de base. O GAP apresentou transição vítrea $(\mathrm{Tg})$ bem definida, que ocorreu a $-49{ }^{\circ} \mathrm{C}$, conforme Figura 4 , valor este muito próximo ao encontrado em literatura ${ }^{[10]}$.

Já o perfil de decomposição da amostra GAP, Figura 5, obtido por meio de análise TGA, mostra que o produto sofreu ignição a $250{ }^{\circ} \mathrm{C}$ na razão de aquecimento de $10{ }^{\circ} \mathrm{C} / \mathrm{min}$ em atmosfera de ar sintético. A maior perda de massa, cerca de $85 \%$, ocorreu entre a temperatura ambiente e $270{ }^{\circ} \mathrm{C}$. A perda de massa restante ocorreu entre 270 e $600{ }^{\circ} \mathrm{C}$, sendo que nesta última temperatura já não havia mais resíduo nessa condição de ensaio.

A análise elementar do GAP apresentou os seguintes valores, de acordo com a Tabela 1.

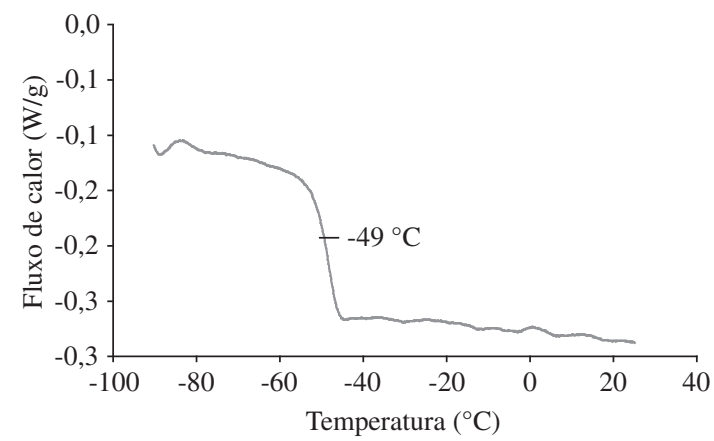

Figura 4. Curva DSC para a amostra de GAP.

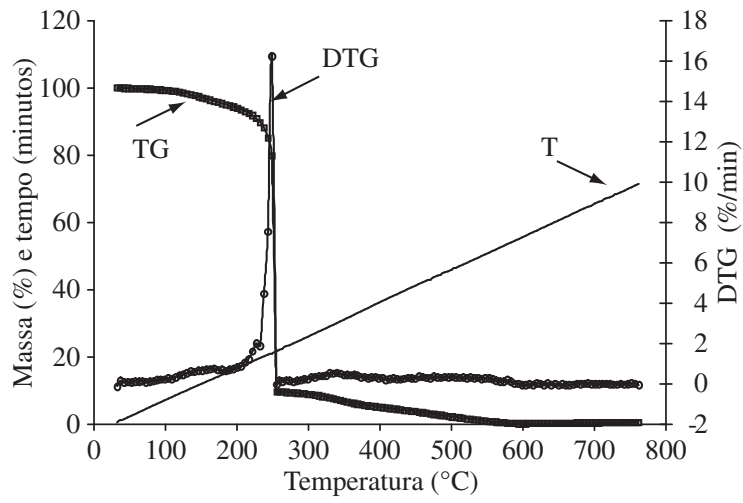

Figura 5. Perfil de decomposição da amostra de GAP por TGA.

Tabela 1. Análise elementar do GAP.

\begin{tabular}{ccc}
\hline Elemento & Valor obtido (\%) & Valor teórico (\%) \\
\hline $\mathrm{C}$ & 36,4 & 36,5 \\
$\mathrm{H}$ & 5,1 & 5,1 \\
$\mathrm{~N}$ & 39,5 & 42,4 \\
\hline
\end{tabular}


Existe uma boa correlação entre os valores obtidos e os teóricos. Pelos valores do nitrogênio pode-se afirmar que houve uma taxa de conversão de poliepicloridrina em GAP de 93\%. Aliás, aqui cabe uma ressalva: provavelmente esta taxa de conversão seja próxima de $100 \%$, uma vez que a análise FTIR não registrou em $748 \mathrm{~cm}^{-1}$ a absorção de grupos $\mathrm{CH}_{2} \mathrm{Cl}$. Então, o que pode ter ocorrido foi a formação de compostos cíclicos $^{[11]}$, que apresentam em sua estrutura carbono e hidrogênio mas, não apresentam grupos $\mathrm{N}_{3}$. Daí os valores obtidos para o carbono e hidrogênio estarem coerentes com o esperado e o do nitrogênio estar um pouco abaixo.

A análise de determinação de massa molar do GAP exibiu como resultado massa molecular média (Mn) de 2725 , massa molecular ponderal média $(\mathrm{Mw})$ de 3128 e dispersividade de 1,15 .

\section{Conclusão}

O polímero glicidil azida (GAP) foi obtido fazendo-se reagir azida de sódio com o intermediário de síntese poliepicloridrina, o qual, por sua vez, foi obtido a partir da homopolimerização da epicloridrina.

Ambos os produtos, final e intermediário de síntese, foram caracterizados por análise FTIR e exibiram espectros bastante semelhantes aos espectros encontrados em literatura.

O GAP foi caracterizado por análises TGA, DSC, elementar e GPC. Apresentou temperatura de Tg à $-49^{\circ} \mathrm{C} \mathrm{e}$ temperatura de ignição em $250{ }^{\circ} \mathrm{C}$. A análise elementar mostrou que os valores obtidos de $\mathrm{C}$ e $\mathrm{H}$ estão dentro dos parâmetros esperados e o de $\mathrm{N}$ ligeiramente abaixo. A massa molecular foi determinada por GPC e apresentou como resultados Mn igual a 2725, Mw igual a 3128 e dispersividade de 1,15 .

\section{Referências Bibliográficas}

1. Klager, K. \& Wrightson, J. M. - "Recent Advances in Solid Propellant Binder Chemistry", in: Mechanics and Chemisty of Solid Propellants, cap.2, Erigem, A. C. (ed.), Pergamon Press, Oxford (1967).

2. Francis, A. U. et al. - Eur. Polym. J., 39, p.831 (2003).

3. Kubota, N. \& Sonobe, T. - "Combustion of GAP Propellants", in: Proceedings of the 19th International Annual Conference of ICT, Karlsruhe, Germany, (1988).

4. Frankel, M. B. et al. - J. Propuls. Power, 8, (3), p.560 (1992).

5. Biedron, T. et al. - J. Polym. Sci., 29, p.619 (1991).

6. Manzara, A. P. - "Primary hydroxyl-terminated polyglycidyl azide”, U.S. patent n 5, 164, p.521 (1992).

7. Brochu, S. \& Ampleman, G. - Macromolecules, 29, p.5539 (1996).

8. Ahad, E. - "Branched Hydroxy Terminated Azido Polymers", in: Proceedings of the $21^{\text {th }}$ International Annual Conference of ICT, Karlsruhe, Germany. (1990).

9. Hummel, D. O. \& Scholl, F. - "Atlas of Polymer and Plastics Analysis”, Verlag Chemie Gmbh D6940, Weinhein (1984).

10. Eroglu, M. S. et al. - Polymer Bulletin, 36, p.695 (1996).

11. Kubisa, P. \& Penczek, S. - Prog. Polym. Sci., 24, p.1409 (1999).

Enviado: $22 / 10 / 08$

Reenviado: $12 / 12 / 08$

Aceito: $15 / 12 / 08$ 